\title{
Impact energy of everyday items used for assault
}

\author{
Lea Siegenthaler $^{1}$ (D) . Florian D. Sprenger ${ }^{1} \cdot$ Beat P. Kneubuehl $^{1} \cdot$ Christian Jackowski $^{1}$
}

Received: 20 March 2017 / Accepted: 6 September 2017

(C) Springer-Verlag GmbH Germany 2017

\begin{abstract}
Blunt force is a frequently used type of violence especially because it can be performed with basically every object of our daily lives or with bare hands or feet. The injuries and medical consequences have been widely examined, whereas the forces and especially the energies acting on impact have rarely been analyzed. The aim of the present study is to provide the impact energy and its ranges of four longish everyday items with different characteristics for male and female offenders. Additionally, the moment of inertia (MOI) for all the objects was calculated and its influence on the energy determined. A combination wrench, aluminum pipe, golf club, and spade were chosen as representatives of the four categories short, medium length with the center of mass $(\mathrm{COM})$ in the middle, medium length with the COM close to the hitting point, and long and heavy. A total of 880 strikes have been performed by 11 volunteers. The results show the mean energy values of wrench, pipe, golf club, and spade for men of 51.1, 74.4, 93.5, and 166.7 J. For women, the results are 33.0, 41.0, 56.5, and 76.8 J. Knowing the energy thresholds for certain fractures and injuries, these results help to assess whether a claimed hit may have caused the fracture or injury or not.
\end{abstract}

Keywords Blunt force trauma $\cdot$ Impact energy $\cdot$ Horizontal striking $\cdot$ Golf club $\cdot$ Spade $\cdot$ Combination wrench . Aluminum pipe

Lea Siegenthaler

lea.siegenthaler@irm.unibe.ch; http://www.irm.unibe.ch

1 Institute of Forensic Medicine, University of Bern, Bern, Switzerland

\section{Introduction}

A considerable part of the daily casework in legal medicine is the analysis of blunt trauma. A significant amount of such cases are strikes with a great variety of longish rigid objects as weapons, which can cause injuries ranging from mild to potentially deadly trauma.

A variety of objects used for hitting can be found in cases reported in the literature emphasizing their injury potential. For instance, a blow to the chest with a golf club, which ended deadly [1], or blows to the eyes, which lead to the loss of eyesight [2]. Baseball bats are dangerous weapons as proven in multiple cases with resulting injuries ranging from bone fractures to deadly injuries to the chest and head [3-8]. The variety of objects used as hitting instruments in forensic casework is infinite, because anything within the perpetrator's reach can be used for this purpose as long as it can be lifted off the ground and accelerated. In the archive of the author's host institute of forensic medicine alone, a multitude of hitting objects can be found, e.g., frying pans, snow shovels, sticks, crowbars, or statues. The present study will only consider longish rigid objects and will disregard objects, which are round or very short and thus cannot be swung, e.g., ashtrays or vases.

Forensic physicists and legal examiners are often asked to classify such attacks for the jurisdiction as mild or strong or to estimate the potential damage an object could cause if a person would be hit with it. Furthermore, the life threatening potential of such a hit is often in question. The impact energy is a good quantity to illustrate the overall dangerousness that can be reached by a certain object. Impact energy, unlike the acting force [9], is independent from the target surface properties and geometry, from the contact area, and from the surface properties and geometry of the hitting object and thus is useful in comparing the hitting objects characteristics. In view of the 
injury risk, the aforementioned quantities cannot totally be neglected; however, for the purpose of this study, only the energy will be regarded. The reason being that the target is mostly unknown since the specific body part to be hit is equally unknown and its properties can merely be estimated. Additionally, the contact area on the object is unknown, considering, e.g., a shovel with either a flat large surface or a very narrow edge.

The target independent properties of the hit were studied in Sprenger et al. [10]. Furthermore, the effective mass of longish rigid objects was studied in Adamec et al. [11]. The hitting energy of a variety of everyday items has so far not been investigated.

The intention of this study was to determine the impact energy and its range of four different everyday items, chosen to represent four categories, i.e., short, medium length with the center of mass (COM) in the middle, medium length with the COM towards the hitting part, and long and heavy. In doing so, the difference between male and female hitters and the inter-individual differences were analyzed.

\section{Materials and methods}

Out of the vast variety of objects used for hitting in forensic cases, an assortment was chosen to represent four categories of instruments. These four objects had different physical properties.

The first category is the one for all short objects, and thus, the COM is located close to the holding hand such as frying pans, glass bottles (that do not break) [12], or candleholders. In this study, they are represented by a combination wrench made of chromed vanadium held at the ring end.

The second category contains objects of medium length with even mass distribution, i.e., the COM is located in the middle including any type of stick, such as broom and shovel handles, square bars, or branches. These instruments are represented by an aluminum pipe having an inner diameter of $21.6 \mathrm{~mm}$ and a wall thickness of $1.5 \mathrm{~mm}$.

The third category includes all objects of medium length with the COM located close to the end furthest away from the perpetrator's hand, here represented by a golf club (MacGregor Golf M565 V-FOIL SPEED, 6 Iron). Sixty percent of the mass is placed in the $50 \mathrm{~mm}$ furthest away.

The fourth category consists of heavy and long objects whose COM usually is not located in their middle because they have a bigger or heavier end used for a specific application. This category contains shovels or hoes and is represented by a spade.

The mass, length, COM, and the moment of inertia (MOI) of the used striking objects are summarized in Table 1. The MOI was calculated assuming a point of rotation at the end held in hands.
Table 1 Properties of the striking objects

\begin{tabular}{lllll}
\hline Object & $m_{\mathrm{o}}(\mathrm{kg})$ & $l_{\mathrm{o}}(\mathrm{m})$ & $\mathrm{COM}(\mathrm{m})$ & $I\left(\mathrm{~kg} \mathrm{~m}^{2}\right)$ \\
\hline Combination wrench & 0.51 & 0.33 & 0.18 & 0.02 \\
Aluminum pipe & 0.3 & 1 & 0.5 & 0.08 \\
Golf club & 0.423 & 1 & 0.725 & 0.22 \\
Spade & 2.205 & 1.17 & 0.79 & 1.38 \\
\hline
\end{tabular}

$m_{\mathrm{o}}$ mass of the striking object, $l_{\mathrm{o}}$ length of the striking object, $C O M$ center of mass measured from the end of the striking object, where it is held, $I$ is the moment of inertia (point of rotation is the end held in hands)

For this study, seven male and four female volunteers in the range of 21 to 29 years of age have been recruited. Physically well-trained as well as non-trained individuals with different professions and leisure activities, from desk workers to a security guard and martial artist participated. The age, height, arm length, perimeter of the upper arm, and leisure activities of the volunteers can be found in Table 2. None of the above properties has been used to in- or exclude volunteers from the study.

In order to measure the striking energies, the volunteers had to hit a pendulum horizontally as hard as they could. The target was located $0.98 \mathrm{~m}$ aboveground and had a diameter of $0.18 \mathrm{~m}$. The mass of the pendulum was $17.3 \mathrm{~kg}$. A test series for one volunteer included 20 strikes with each object, resulting in 80 measurements per volunteer. A total of 880 measurements have been acquired.

The target on the pendulum was covered with modeling clay to achieve an inelastic collision. The angular movement of the pendulum was measured with a potentiometer connected to an oscilloscope (Picoscope 5203 PC oscilloscope). To determine the striking velocity of the impacting part, each strike was filmed with a high-speed camera (Casio Exilim High Speed EX-FC 100) at 420 fps vertically from below the pendulum.

A damped sinusoidal wave was fitted to match the curves of the oscilloscope with "Matlab." The greatest gradient was then calculated with the first derivative of the sinus curve, which corresponds to the greatest angular velocity of the pendulum.

The kinetic energy of the strike cannot be measured directly in the setting of an inelastic collision. It was determined by the impact velocity of the striking object onto the pendulum measured at the point of impact and the fictive impact mass (defined as the point mass that would produce the same impact energy as the striking object acting in the moment of impact). This impact mass consists of a part of the mass of the striking object and parts of the arm and upper body of the volunteer, which is why it cannot be measured directly. This allows only a calculation by means of the law of conservation of momentum using 
Table 2 Description of the volunteers

\begin{tabular}{lllllll}
\hline & Age (years) & Height $(\mathrm{cm})$ & Mass $(\mathrm{kg})$ & Arm length $(\mathrm{cm})$ & $\begin{array}{l}\text { Perimeter upper } \\
\text { arm }(\mathrm{cm})\end{array}$ \\
\hline Vm 1 & 25 & 178 & 68 & 81 & 31 & Sports \\
Vm 2 & 24 & 183 & 87 & 84 & 36 & Fencing, floorball \\
Vm 3 & 22 & 193 & 77 & 83 & 31 & Noxing \\
Vm 4 & 22 & 192 & 86 & 81 & 37 & None \\
Vm 5 & 29 & 185 & 91 & 84 & 34 & Muay Thai, soccer \\
Vm 6 & 26 & 181 & 84 & 83 & 30 & Soccer \\
Vm 7 & 25 & 195 & 72 & 88 & 29 & Bicycle racing \\
Vf 1 & 21 & 164 & 62 & 73 & 28 & Dancing \\
Vf 2 & 26 & 177 & 64 & 80 & 26 & None \\
Vf 3 & 24 & 164 & 74 & 78 & 32 & Fencing, climbing \\
Vf 4 & 24 & 173 & 74 & None \\
\hline
\end{tabular}

$V m$ refers to the male volunteers and $v f$ to the female volunteers. The arm length was measured from acromion to fingertip of the middle finger while hanging, the perimeter of the hitting upper arm with tense muscles

$m_{\mathrm{s}} \cdot v_{\mathrm{s}}=\left(m_{\mathrm{p}}+m_{\mathrm{s}}\right) \cdot v_{\max }$

where $m_{\mathrm{s}}$ denotes the impact mass, $v_{\mathrm{s}}$ is the impact velocity of the striking object, $m_{\mathrm{p}}$ is the mass of the pendulum, and $v_{\max }$ is the maximum trajectory velocity of the pendulum in its COM. The formula was then transposed to the following:

$m_{\mathrm{s}}=\frac{m_{\mathrm{p}} \cdot v_{\max }}{v_{\mathrm{s}}-v_{\max }}$

to calculate the impact mass, with the impact velocity measured by high-speed camera and the calculated impact mass, the impact energy was obtained [10].

A two-sample Student's $t$ test assuming unequal variances with a significance level of 0.05 was used to analyze the differences between men and women.

\section{Results}

The average of the mean hitting energies, mean hitting velocities, and mean fictive impact masses and their respective ranges are listed in Table 3 for the male volunteers and in Table 4 for the female volunteers.
Taking into account, all the individual strikes performed by all the volunteers, the mean energies, velocities, and masses of the male and female hitters were compared. As expected, the energies of the male hitters were all significantly higher than the values of the females with regard to a significance level of 0.05 . This applies also to the velocity, but the reverse is true for the impacting mass. Apart from the value for the spade, the values of the females are higher. The values for the wrench and golf club are even significantly higher than the male values.

The inter-individual difference is shown in Fig. 1, where the mean impact energies of the male (vm) and the female volunteers (vf) for each object are arranged in increasing order. Additionally, the standard deviation for each volunteer is displayed.

For the wrench, the female volunteer with the highest mean hitting energy exceeded the energy of the weakest male volunteer. Three of four female hitters achieved mean energies within the standard deviation of the male volunteers.

Using the aluminum pipe, only one female hitter reached a mean value lying within the standard deviation of the male hitters. This value exceeded that of the other female volunteers by far and was close to that of the weakest male hitter.
Table 3 Physical characteristics of the hits performed by the male volunteers

\begin{tabular}{llll}
\hline Men & $E_{\mathrm{i}}(\mathrm{J})$ & $v_{\mathrm{s}}(\mathrm{m} / \mathrm{s})$ & $m_{\mathrm{s}}(\mathrm{kg})$ \\
\hline Combination wrench & $51.1(35.1-65.8)$ & $20.7(18.5-24.1)$ & $0.238(0.205-0.312)$ \\
Aluminum pipe & $74.4(57.4-84.9)$ & $31.1(27.7-36.2)$ & $0.156(0.129-0.190)$ \\
Golf club & $93.5(59.8-105.7)$ & $24.3(18.7-28.1)$ & $0.317(0.266-0.343)$ \\
Spade & $166.7(107.2-217.8)$ & $14.3(11.6-16.8)$ & $1.622(1.402-1.842)$ \\
\hline
\end{tabular}

$E_{\mathrm{i}}$ mean impact energy, $v_{\mathrm{s}}$ mean impact velocity of the striking object, $m_{\mathrm{s}}$ mean impact mass of the strike. In brackets, the range of the individual mean values 
Table 4 Physical characteristics of the hits performed by the female volunteers

\begin{tabular}{llll}
\hline Women & $E_{\mathrm{i}}(\mathrm{J})$ & $v_{\mathrm{s}}(\mathrm{m} / \mathrm{s})$ & $m_{\mathrm{s}}(\mathrm{kg})$ \\
\hline Combination wrench & $33.0(32.1-35.7)$ & $15.2(13.7-16.0)$ & $0.288(0.256-0.345)$ \\
Aluminum pipe & $41.0(33.7-56.5)$ & $22.8(19.6-27.3)$ & $0.158(0.145-0.177)$ \\
Golf club & $56.5(46.4-75.3)$ & $18.3(15.9-21.8)$ & $0.339(0.307-0.366)$ \\
Spade & $76.8(42.4-98.8)$ & $10.5(8.5-11.6)$ & $1.364(1.152-1.571)$ \\
\hline
\end{tabular}

$E_{\mathrm{i}}$ mean impact energy, $v_{\mathrm{s}}$ mean impact velocity of the striking object, $m_{\mathrm{s}}$ mean impact mass of the strike. In brackets, the range of the individual mean values
Hitting with the golf club, the female volunteer with the highest mean energy exceeded the energy of the weakest male volunteer. Three of four female hitters reached values within the standard deviation of the male volunteers.

The measurements with the spade resulted in two out of four female hitters achieving values within the standard deviation of the male volunteers.

For every instrument, individual energy values of all the female hitters lay within the standard deviation of all the males.

In Figs. 2 and 3, the frequency distributions of all the single strikes of the different striking objects are shown. All the male hitters together (Fig. 2) executed a total of 140 hits with each object whereas the female ones (Fig. 3) executed 80. The energies were divided into intervals of $10 \mathrm{~J}$. The highest measured energy value of a male hitter was $289 \mathrm{~J}$ and that of a female $158 \mathrm{~J}$.

Furthermore, the influence of the MOI on the hitting energy was considered. A fit was performed based on the following theoretical formula used to calculate the energy of an object thrown by hand [13]:

$E_{\text {ini }}=\frac{m_{\mathrm{object}}}{m_{\mathrm{object}}+m_{\mathrm{arm}}} \cdot W$ where $E_{\text {ini }}$ is the initial energy of the thrown object, $m_{\text {object }}$ is the mass of the thrown object, $m_{\mathrm{arm}}$ is the mass of the arm, and $W$ is the work performed by the thrower.

This formula was converted to fit a motion with a striking instrument opposed to a thrown object; thus, the masses were changed to MOI. The MOI of each instrument can be found in Table 1, but it would not suffice to simply exchange the masses with these MOIs since the point of rotation is not the handle of the instrument but the torso or the shoulder joint. Therefore, a correctional term is needed to be added compensating the too small MOI of the hitting instrument leading to the following formula:

$E_{\text {imp }}=\frac{I_{\text {inst }}+I_{\text {cor }}}{I_{\text {inst }}+I_{\text {cor }}+I_{\text {arm }}} \cdot W$

where $E_{\text {imp }}$ is the impact energy, $I_{\text {arm }}$ is the MOI of the arm, $I_{\text {inst }}$ is the MOI of the hitting instrument, $I_{\text {cor }}$ is the correctional term, and $W$ is the work the hitter puts into his motion. Measurements provided the values of the impact energy and the MOI of the hitting instrument while the rest of the parameters were fitted. The corresponding values can be found in Table 5.

The correctional term was used to calculate the actual MOI influencing the impact $\left(I_{\text {tot }}=I_{\text {inst }}+I_{\text {cor }}\right)$. The impact energy as
Fig. 1 Mean impact energy and standard deviation of all subjects and all objects. The energies of the male volunteers $(\mathrm{vm})$ and the female volunteers (vf) are grouped separately and arranged in increasing order for each object

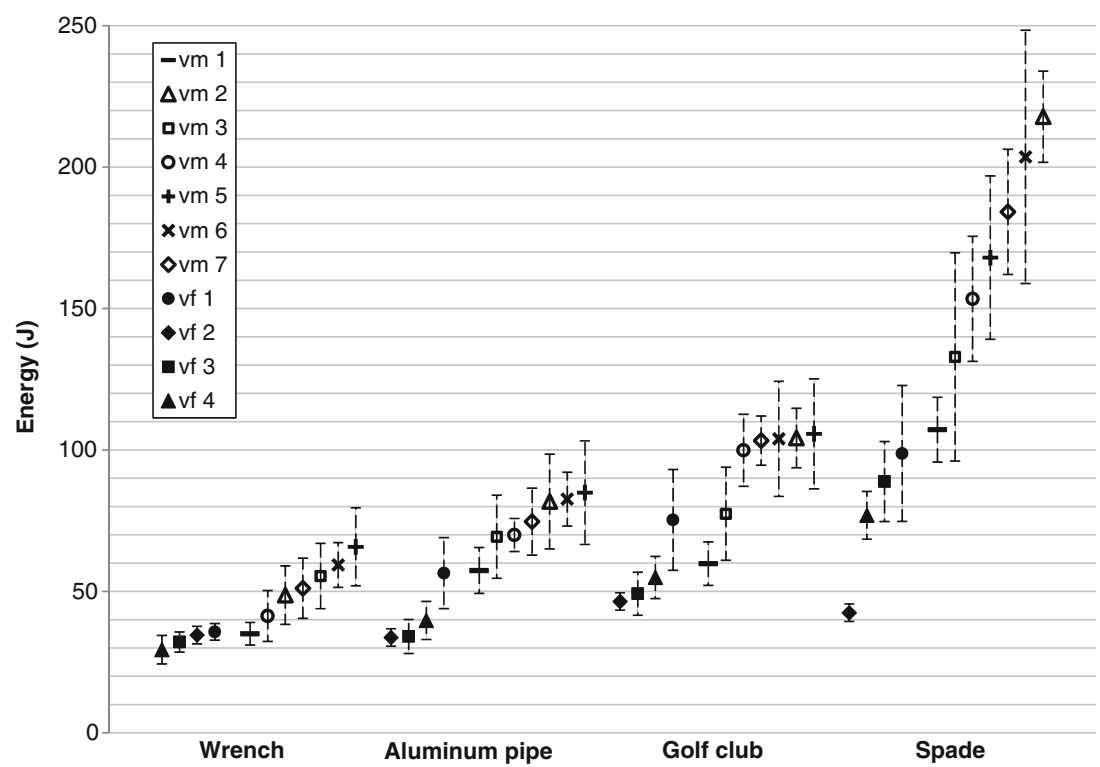


Fig. 2 Frequency distribution of the male subjects for the striking energies of all objects. One hundred forty hits were executed with each object. The impact energies were divided into intervals of $10 \mathrm{~J}$. No energies higher than $300 \mathrm{~J}$ have been measured for these objects

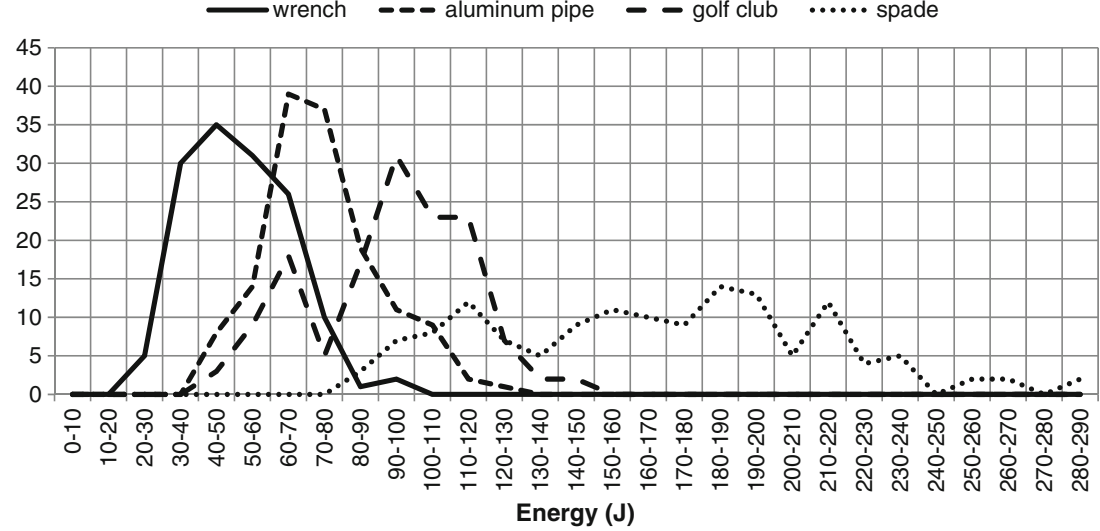

a function of the actual MOI and the corresponding fit can be seen in Fig. 4.

The influence of the body parameters was also taken into consideration. The influence of the parameter with the best correlation, which is the body mass, is shown in Fig. 5 with the corresponding trend line and coefficient of determination for each object.

\section{Discussion}

Comparing both genders, it becomes apparent that the biggest difference is found for the heaviest hitting instrument, the spade, where the mean value is higher by a factor of 2.2 for the male hitters. This can be explained by the fact that for some women, its mass was close to the maximum possible mass that a hitting instrument can have due to their physiological limits. In contrast, the mean values of the male hitters were higher by a factor of 1.6 for the wrench, 1.8 for the pipe, and 1.7 for the golf club, respectively.

Due to the greater influence of the impact velocity than that of the impact mass on the impact energy, the higher impact masses of the women had a smaller effect than the higher impact velocities of the men leading to the higher energies of the male volunteers. An explanation for the higher impact masses of the women has so far not been found.

For both female and male hitters, the energy values of the spade had the widest spread distribution as can be seen in Figs. 2 and 3 whereas the wrench has the narrowest, albeit not by far. Note that the number of hits per instrument differs in the two figures because of the different number of volunteers, resulting in 140 hits for the seven male volunteers and 80 hits for the four female volunteers.

The comparison between the aluminum pipe and the golf club shows the influence of a difference in the COM of $22.5 \mathrm{~cm}$ corresponding to $22.5 \%$ of the total length. This leads to a $20.4 \%$ increase in the energy for the men and $27.4 \%$ for the women, respectively. Unfortunately, the masses of the two objects could not have been chosen to be completely identical, which means the influence of the COM cannot be totally isolated. Instead it makes more sense to regard the influence of the MOI diagramed in Fig. 4, where the influence of the arm swinging the instrument is taken into account. It can be seen that a higher MOI results in a higher hitting energy, up to a certain point, which was not reached by the used objects. Therefore, the highest possible MOI could not be determined.

The correlation between the body parameter and the energy was better than expected but can hardly be used to make predictions about the energy produced by a certain person.
Fig. 3 Frequency distribution of the female subjects for the striking energies of all objects. Eighty hits were executed with each object. The impact energies were divided into intervals of $10 \mathrm{~J}$. No energies higher than $170 \mathrm{~J}$ have been measured for these objects

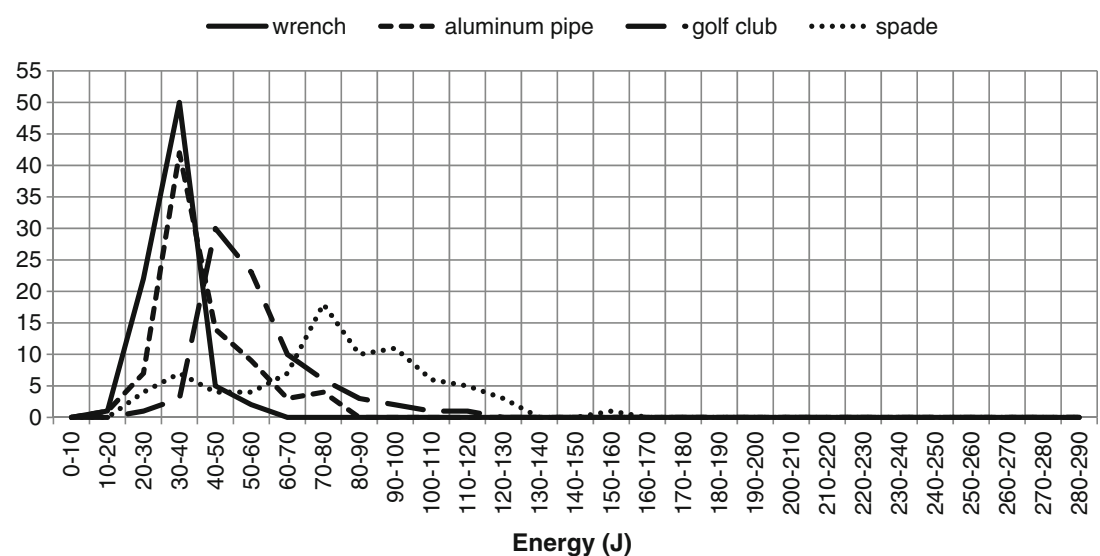


Table 5 The fitting parameters for the male and female volunteers

\begin{tabular}{lll}
\hline Parameter & Men & Women \\
\hline$I_{\text {arm }}$ & 0.44 & 0.17 \\
$I_{\text {cor }}$ & 0.13 & 0.08 \\
$W$ & 214.72 & 85.65 \\
\hline
\end{tabular}

$I_{\text {arm }}$ is the mean moment of inertia of the arm, $I_{\text {cor }}$ is a correctional term, and $W$ is the work the hitter puts into his motion

Furthermore, the measured impact energy can be compared to literature findings of energy values causing thorax and head injuries. For example, rubber projectiles weighing $140 \mathrm{~g}$ were shot at the thorax and abdomen of anesthetized pigs with speeds ranging from 30 to $64 \mathrm{~m} / \mathrm{s}$ resulting in energies from 63.0 to $286.7 \mathrm{~J}$. Cardiac compression and contusion, and rib fractures as well as vessel ruptures were observed [14]. Another energy threshold for the thorax is given through the testing standard of ballistic body armor where an energy transfer to the body of $70 \mathrm{~J}$ is allowed [15]. Thresholds for skull fractures were investigated in multiple studies. For instance, fractures of the neurocranium with an electrohydraulic device and unembalmed intact human cadaver heads were measured. The failure loads depended on the anatomical localization and ranged from 14.1 to $68.5 \mathrm{~J}$ [16]. Hard projectiles were shot in the face of post-mortem human subjects with energies between 14 and $84 \mathrm{~J}$ to the forehead and 16 to $26 \mathrm{~J}$ on the mandible, which did not result in fractures, whereas 10-50 J on the zygoma always resulted in a fracture [17]. The temporo-parietal resistance of the head on unembalmed postmortem human subjects was tested in a free-falling drop test. Fractures occurred between 16 and $53 \mathrm{~J}$ [18]. With porcine cranium, a test was performed resembling a hammer strike: Fractures were found for energies higher than $18 \mathrm{~J}$ [19].

The values of the impact energies elaborated in this study show that all the male volunteers and stronger female

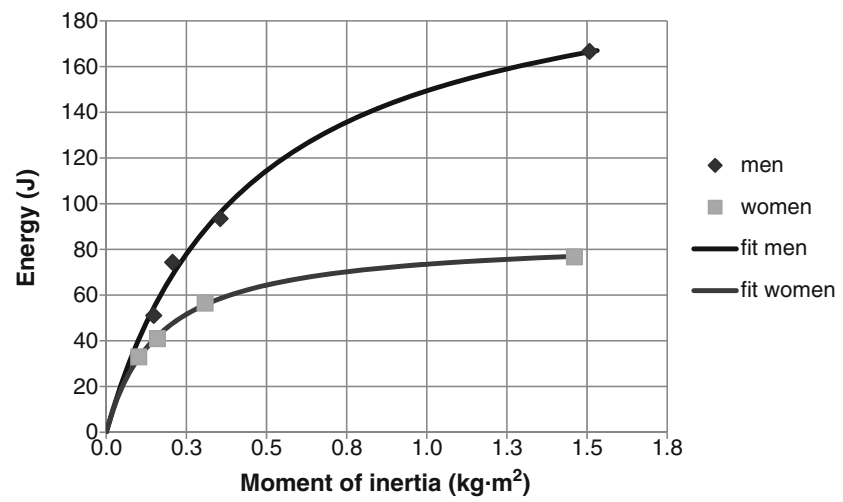

Fig. 4 The influence of the moment of inertia of the hitting instrument held by hand. The mean energy for each object as a function of the total MOI of the arm and the hitting instrument is displayed. A fit was calculated based on Eq. (4)

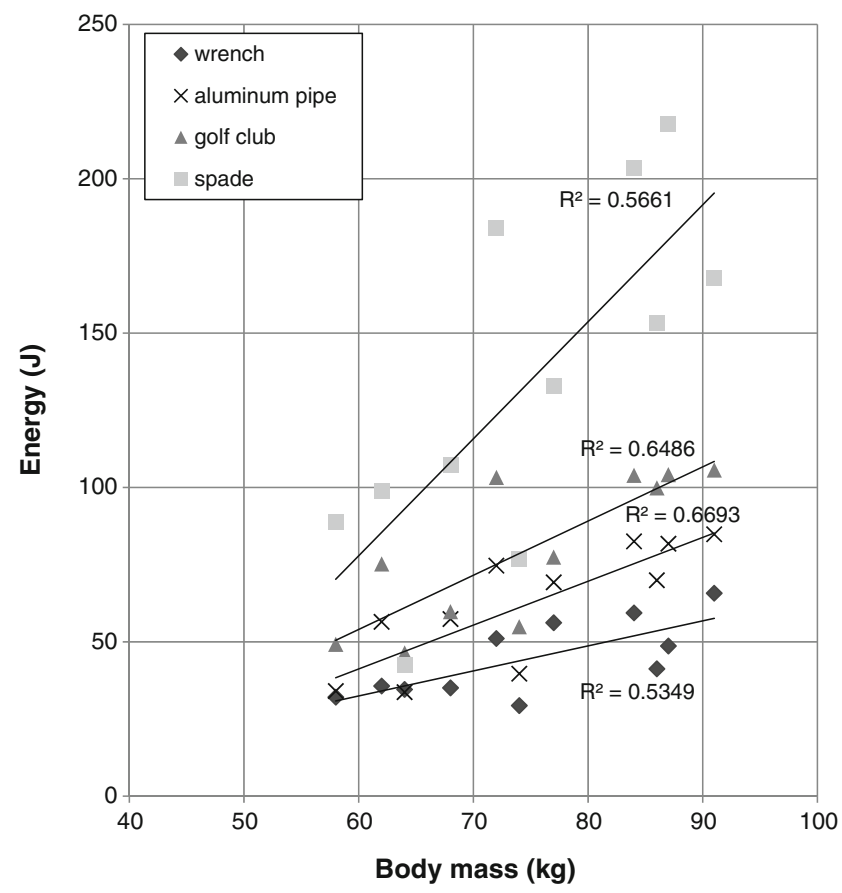

Fig. 5 The influence of the body mass on the energy. The linear trend line is shown with the corresponding coefficient of determination for each object

volunteers would be able to inflict severe injuries such as rib fractures and organ or vessel lacerations.

\section{Limitations}

The results of this study are subject to a number of uncertainties due to the experimental setup. If the target is not hit in the center and in the movement axis of the pendulum, some of the energy is transformed in vibration instead of being transmitted into the movement of the pendulum. The calculations assume an absolute inelastic collision, which in reality can only approximately be reached.

The statistical conclusions would be more meaningful with a greater number of participants.

Throughout the experiment, the hitters became accustomed to the previously unknown movement, thus improving the aim, which resulted in higher energy values. A different design of the pendulum, which could measure the impact energy independently of the striking direction and with a bigger target area, could decrease this effect. Furthermore, due to the strenuous task, the untrained individuals tired faster influencing the average result.

An additional source of error was the analysis of the highspeed videos. At a framerate of $420 \mathrm{fps}$, the resolution of the pictures was limited and light effects could overlay the markings on the striking objects, which could cause small inaccuracies in the evaluation process. The distance between the impactor and the camera differed slightly in each strike, and 
therefore, the scale, which has been centered on the target, was not perfectly accurate for every hit.

The study was limited to horizontal strikes, and it is likely that vertical or diagonal strikes would lead to different results. These types of strikes should also be investigated but would require a different setup.

\section{Conclusion}

This study provides the impact energies, impact masses, and impact velocities for horizontal strikes with a combination wrench, an aluminum pipe, a golf club, and a spade, as representatives for everyday items. Additionally, it compares the ranges of all the objects for female and male volunteers. The higher the MOI, the higher is the energy, up to the point where the hitter is unable to hold or accelerate the object. The energy and velocity of all objects are significantly higher for the male volunteers than they are for female volunteers. However, the impact masses of the female hitters are higher except for the spade. The reason is so far unclear. There is a correlation between the body mass and the hitting energy. Literature values for skull fractures and thorax injuries can be consulted, in order to determine the injury potential of hits with each object.

Acknowledgements All procedures performed in studies involving human participants were in accordance with the ethical standards of the institutional and/or national research committee and with the 1964 Helsinki declaration and its later amendments or comparable ethical standards. Informed consent was obtained from all individual participants included in the study.

\section{Compliance with ethical standards}

Conflict of interest The authors declare that they have no conflict of interest.

\section{References}

1. Purdue B, Fernando GC (1989) The mechanism of fatal cardiopulmonary injury caused by a blow from a golf club. Forensic Sci Int 42(1-2):125-130

2. Jayasundera T, Vote B, Joondeph B (2003) Golf-related ocular injuries. Clin Experiment Ophthalmol 31(2):110-113
3. Groleau GA, Tso EL, Olshaker JS, Barish RA, Lyston DJ (1993) Baseball bat assault injuries. J Trauma 34(3):366-372

4. Ord R, Benian R (1995) Baseball bat injuries to the maxillofacial region caused by assault. J Oral Maxillofac Surg 53(5):514-517. https://doi.org/10.1016/0278-2391(95)90061-6

5. Gläser N, Kneubuehl BP, Zuber S, Axmann S, Ketterer T, Thali MJ, Bolliger SA (2011) Biomechanical examination of blunt trauma due to baseball bat blows to the head. J Forensic Biomech 2 . https://doi.org/10.4303/jfb/F100601

6. David C, Viano DVA (1992) Fatal chest injury by baseball impact in children: a brief review. Clin J Sport Med 2(3). https://doi.org/10. 1097/00042752-199207000-00002

7. Bryant D, Greenfield R, Martin E (1992) Musculoskeletal trauma- the baseball bat. J Natl Med Assoc 84(11):957-960

8. Levy AS, Bromberg J, Jasper D (1994) Tibia fractures produced from the impact of a baseball bat. J Orthop Trauma 8(2):154-158

9. Sulaiman NA, Osman K, Hamzah NH, Amir SP (2014) Blunt force trauma to skull with various instruments. Malays J Pathol 36(1):3339

10. Sprenger FD, Siegenthaler L, Kneubuehl BP, Jackowski C (2015) The influence of striking object characteristics on the impact energy. Int J Legal Med:1-10. https://doi.org/10.1007/s00414-0151268-1

11. Adamec J, Praxl N, Schneider K, Graw M (2011) Estimation of effective mass of longish rigid instruments in head impacts. Int $\mathrm{J}$ Legal Med 125(6):763-771. https://doi.org/10.1007/s00414-0100490-0

12. Bolliger SA, Ross S, Oesterhelweg L, Thali MJ, Kneubuehl BP (2009) Are full or empty beer bottles sturdier and does their fracture-threshold suffice to break the human skull? J Forensic Legal Med 16(3):138-142. https://doi.org/10.1016/j.jflm.2008.07. 013

13. Kneubuehl BP (2013) Geschosse - Gesamtausgabe, BallistikMesstechnik-Wirksamkeit-Treffsicherheit, 2013th edn. Motorbuch Verlag, Stuttgart

14. Cooper GJ, Taylor DE (1989) Biophysics of impact injury to the chest and abdomen. J R Army Med Corps 135(2):58-67

15. Vereinigung der Prüfstellen für angriffshemmende MuK (2008) PRÜFRICHTLINIE "Ballistische Schutzwesten". Vereinigung der Prüfstellen für angriffshemmende Materialien und Konstruktionen (VPAM)

16. Yoganandan N, Pintar FA, Sances A Jr, Walsh PR, Ewing CL, Thomas DJ, Snyder RG (1995) Biomechanics of skull fracture. J Neurotrauma 12(4):659-668

17. Viano DC, Bir C, Walilko T, Sherman D (2004) Ballistic impact to the forehead, zygoma, and mandible: comparison of human and frangible dummy face biomechanics. J Trauma 56(6):1305-1311

18. Yoganandan N, Zhang J, Pintar F (2004) Force and acceleration corridors from lateral head impact. Traffic Inj Prev 5(4):368-373. https://doi.org/10.1080/15389580490510336

19. Mole CG, Heyns M, Cloete T (2015) How hard is hard enough? An investigation of the force associated with lateral blunt force trauma to the porcine cranium. Legal Med 17(1):1-8. https://doi.org/10. 1016/j.legalmed.2014.07.008 